\section{PSICOLOGÍA IBEROAMERICANA}

\section{Psicología Iberoamericana}

ISSN: 1405-0943

psicología.iberoamericana@uia.mx

Universidad Iberoamericana, Ciudad de

México

México

Valdés Cuervo, Ángel Alberto; Bautista Hernández, Gildardo; Vera Noriega, José Ángel; Herrera Mateos, Juan Carlos

Variables que diferencian a estudiantes de secundarias con y sin reportes de bullying en la escuela

Psicología Iberoamericana, vol. 21, núm. 1, enero-junio, 2013, pp. 32-41

Universidad Iberoamericana, Ciudad de México

Distrito Federal, México

Disponible en: http://www.redalyc.org/articulo.oa?id=133929862005

- Cómo citar el artículo

Número completo

- Más información del artículo

Página de la revista en redalyc.org

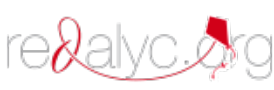

Sistema de Información Científica

Red de Revistas Científicas de América Latina, el Caribe, España y Portugal Proyecto académico sin fines de lucro, desarrollado bajo la iniciativa de acceso abierto 


\title{
Variables que diferencian a estudiantes de secundarias con y sin reportes de bullying en la escuela
}

\author{
Variables that differentiate Junior High School Students \\ with and without Bullying in School
}

\author{
Ángel Alberto Valdés Cuervo* \\ Instituto TECNOLÓGICO de SONORA \\ Gildardo Bautista Hernández \\ José Ángel Vera Noriega \\ Centro de Investigación en Alimentación y Desarrollo A.C. \\ Juan Carlos Herrera Mateos \\ Universidad Pedagógica Nacional de Nogales, Sonora
}

\section{RESUMEN}

El estudio se propuso determinar las diferencias entre estudiantes de secundarias, con y sin reportes de bullying, en variables personales (ajuste escolar y psicosocial) y familiares (composición familiar, ajuste psicosocial y clima familiar). Se realizó un estudio cuantitativo con diseño comparativo, para lo cual se seleccionaron dos grupos de 81 alumnos con y sin reportes de bullying, a los cuales se les administraron los cuestionarios de Caracterización de la violencia escolar y de Percepción del clima familiar. Se apreció que el grupo de estudiantes que realizan bullying presentan menor ajuste escolar y psicosocial, provienen con mayor frecuencia de familias diferentes a la nuclear, con dificultades de ajuste psicosocial y con un clima familiar conflictivo. Se concluyó que los resultados fundamentan la necesidad de un enfoque ecológico en la prevención del bullying que incluya, entre otros, aspectos como intervenciones a nivel individual y familiar.

Palabras clave: violencia escolar, bullying, factores de riesgo, ajuste psicosocial, familia.

\section{ABSTRACT}

The study aimed to determine the differences between junior high school students with and without bullying in personal variables (school adjustment and psychosocial) and family (family composition, psychosocial adjustment and family environment). We performed a quantitative study with comparative design, for which two groups of 81 students with and without reports of bullying were selected respectively, to which questionnaires of "Characterization of school violence" and family atmosphere were administered. It was observed that student with bullying have lower school and psychosocial adjustment, frequently these student come from families different to the nuclear, with psychosocial adjustment and conflict in their family atmosphere. It was concluded that the results support the need for an ecological approach to the prevention of bullying, including among others aspects interventions at the individual and family level.

Keywords: school violence, bullying, risk factors, psychosocial adjustment, family.

* Recibido: 8 de noviembre, 2012 - Aceptado: 23 de marzo, 2013

Correspondencia: Ángel Alberto Valdés Cuervo, Departamento de Educación, Instituto Tecnológico de Sonora.

e-mail: angel.valdes@itson.edu.mx 


\section{INTRODUCCIÓN}

El presente trabajo realiza una investigación acerca de la violencia entre pares que se presenta dentro del contexto escolar, que en su grado más extremo se define como bullying. Se caracteriza por tres particularidades que lo identifican y diferencian de otros tipos de conductas violentas entre pares: a) un uso deliberado de la agresión con la intención de causar daño físico y/o psicológico, b) una relación desigual de poder entre agresor-víctima y c) carácter repetitivo (Coloroso, 2004; Olweus, 1993).

En los últimos años se ha observado en México un renovado interés por el estudio y la atención de las distintas manifestaciones del bullying, a lo que ha contribuido el creciente número de estudios que evidencian la presencia de este fenómeno en las escuelas mexicanas. Dentro de este grupo de trabajos se encuentran los de Avilés \& Monjas (2005), quienes hallaron que 11.6\% de los estudiantes de educación básica reconocen haber estado envueltos en situaciones de maltrato como víctimas y $5.7 \%$ como agresores.

Asimismo, el Instituto Nacional de Evaluación Educativa (INEE, 2006) encontró que el 11\% de alumnos de primarias y secundarias refieren haber participado en peleas, $43.6 \%$ comentan que en las escuelas les han robado, $14 \%$ haber sido lastimados por otros alumnos y 13.6\% recibir burlas de los compañeros; Castillo \& Pacheco (2008) reportaron que 52.8\% de los estudiantes de secundaria afirmaron que les han puesto apodos ofensivos, $48.8 \%$ haber sido víctimas de insultos, $22.2 \%$ de golpes y $22.9 \%$ de exclusión social. Por su parte, Valdés et al. (2012) encontraron que 20.6\% de estudiantes de secundaria reconocen llevar a cabo conductas violentas en la escuela contra los compañeros, e incluso $18.5 \%$ con respecto a los profesores.

El bullying se origina en la interacción de múltiples factores, lo que implica que para la adecuada comprensión de este fenómeno se debe partir de un enfoque ecológico, que impone la consideración de elementos de riesgo situados en la historia, tanto del individuo como de las interacciones que establece dentro de los diferentes contextos donde se desarrolla: comunidad, familia y escuela (Stephenson \& Smith, 2008; Swearer, Espegale \& Napolitano, 2009).

Atendiendo a la necesidad de enfocar el bullying desde una perspectiva ecológica, este estudio preten- dió determinar si aspectos relativos a los propios estudiantes (ajuste escolar y riesgo psicosocial) y a las familias (composición familiar, ajuste psicosocial de los padres y clima familiar) diferenciaban a estudiantes de secundaria con reportes de bullying de aquellos que no manifiestan tales conductas.

Es necesario puntualizar que se identificaron como bullies a aquellos estudiantes que reportaron llevar a cabo conductas agresivas hacia los pares, ya fuera de tipo físico, psicológico o social, dos o más veces al mes (Cassidy, 2009; Olweus, 1993). Se consideró que el ajuste escolar comprende comportamientos que sugieren el cumplimiento por parte del estudiante de las demandas de la escuela, y se consideraron como variables indicadoras del mismo el rendimiento académico y los reportes por problemas de disciplina (Bringas, Rodríguez \& Herrero, 2009; White \& Loeber, 2008). Con respecto a estas variables se elaboraron dos hipótesis de trabajo: a) el rendimiento académico del grupo de estudiantes con reportes de bullying es significativamente menor que el del grupo que no manifiesta esta conducta; b) el grupo de estudiantes con reportes de bullying presentaba de manera significativa mayor proporción de estudiantes con problemas de disciplina.

El riesgo psicosocial integra conductas que aumentan la vulnerabilidad del estudiante a presentar problemas psicológicos o de adaptación social. En este caso se tomaron como indicadores operacionales del mismo el consumo de sustancias, la pertenencia a bandas juveniles o la posesión de amistades cercanas con estos comportamientos (Morales, 2008; Sánchez \& Valdés, 2003). La hipótesis de investigación con respecto a estas variables sostuvo que el grupo de estudiantes que realiza bullying presenta de manera significativa: a) una frecuencia de consumo de alcohol y drogas, b) mayor proporción estudiantes que pertenece a bandas juveniles, c) mayor frecuencia de consumo de sustancias entre los amigos cercanos y d) mayor proporción de amigos que pertenece a bandas.

En lo relativo a la familia se consideró el ajuste psicosocial de la misma, que comprende características y formas de funcionamiento que se asocian a una mejor respuesta a las necesidades de sus integrantes y demandas de su contextos de desarrollo. En este caso se consideraron como factores de riesgo una estructura familiar diferente a la nuclear, la presencia de abuso de sus- 
tancias y de problemas legales en los padres, y un clima familiar negativo. Con respecto a estas variables se realizaron las siguientes hipótesis: a) la proporción de familias diferentes a la nuclear es mayor en el grupo de estudiantes que reportaron realizar bullying, b) la frecuencia de consumo de alcohol y drogas es mayor en los padres en el grupo que refirió efectuar bullying, c) la proporción de familias que presenta problemas legales es mayor en el grupo que mencionó realizar bullying y d) existe un clima familiar más positivo en el grupo de estudiantes que no comete bullying (Eiden et al., 2010; Kelly \& Emery, 2003; Valdés, 2010).

La información que se obtuvo de este estudio permitirá ampliar la comprensión teórica de los factores de riesgo involucrados en la presencia de bullying en estudiantes mexicanos, además, contribuirá a orientar los esfuerzos de los programas de intervención con relación a este fenómeno. El hecho de que las intervenciones se basen en el conocimiento generado acerca de la naturaleza y los factores de riesgo de los fenómenos que procuran eliminar o disminuir, se asocia a un incremento considerable de los alcances y la eficiencia de los mismos (McWhirter, McWhirter, McWhirter \& McWhirter, 1993).

\section{Factores del propio estudiante asociados a la presencia de bullying}

Diversos estudios reportan que los estudiantes que cometen bullying se caracterizan por tener una historia de problemas de ajuste escolar que se manifiestan en un bajo desempeño, pobres expectativas académicas (Muñoz, 2008; Nansel et al., 2001; Ozer, Tarik \& Atik, 2011; Villareal, Sánchez \& Gonzalo, 2010) y problemas de disciplina en la escuela, los cuales se expresan en características como impulsividad, dificultades para seguir las normas escolares, relaciones problemáticas con los demás estudiantes e incluso con los propios profesores (Cava, Musitu \& Murgui, 2006; Díaz, 2005; Gentile, Coyne \& Walsh, 2011).

Es común también que los estudiantes que actúan con violencia hacia los pares presenten dificultades de ajuste psicosocial, ya que tienden a involucrarse con mayor frecuencia que el resto de sus compañeros en conductas antisociales y abuso de sustancias (Jankauskiene, Kardelis, Sukys \& Kardeliene, 2008; Turagabeci, Nakamura \& Takano, 2008).

\section{Factores familiares asociados a la presencia} de bullying

Un grupo importante de estudios refiere que el bullying se asocia a características del contexto familiar que impiden u obstaculizan el desarrollo de competencias académicas y sociales en los hijos. Se han señalado aspectos relativos a dificultades en el ajuste psicosocial de los propios padres, que presentan conductas tales como abuso de sustancias, entre otras, que les ocasionan en muchos casos, inclusive, problemas legales (Eiden et al., 2010; Valdés, Ojeda, Urías \& Ponce, en prensa).

Otro grupo de factores es el de aquellos que afectan directa o indirectamente, de manera negativa, el funcionamiento y por lo tanto el desarrollo de los hijos. Estos se relacionan con una escasa supervisión y participación de los padres en la educación (Ahmed \& Braithwaite, 2004), dificultades para enseñar límites, permisividad ante las conductas violentas (Besag, 2008; Díaz, 2005), uso de métodos violentos para ejercer la autoridad y finalmente modelos paternos disfuncionales (Estévez, Martínez, Moreno \& Musitu, 2006; Olweus, 1993).

Se ha señalado de igual manera que en las familias de los menores con conductas violentas hay un clima familiar conflictivo que ocasiona dificultades en la comunicación, escasa disponibilidad y apoyo por parte los padres. Esto aumenta la probabilidad de la violencia en los hijos, al generar un bajo autoconcepto y menosprecio por las normas escolares, la autoridad de los docentes y los sentimientos de los compañeros (Cava et al., 2006; Díaz, 2005; Estévez et al., 2006; Estévez, Murgui, Moreno \& Musitu, 2007).

\section{MÉTODO}

En términos metodológicos, se trató de un estudio cuantitativo con un diseño comparativo y transversal.

\section{Participantes}

Participaron en el estudio 11 escuelas secundarias públicas del noroeste del estado de Sonora. En una primera etapa, mediante un muestreo aleatorio, tomando como base una probabilidad de éxito del 50\% y un nivel de confianza del 95\% ( $\mathrm{p}=.50 ; \mathrm{q}=.5)$ se seleccionaron 900 alumnos.

En un segundo momento se identificaron a 81 (9\%) estudiantes que realizaban bullying, ya que repor- 
taron llevar a cabo dos o más veces en un mes conductas violentas hacia los compañeros (Cassidy, 2009; Olweus, 1993). Posteriormente, para efectos de la comparación, se seleccionó de manera aleatoria a un subgrupo de 81 estudiantes de los 819 que no fueron identificados como agresores. Esto permitió que se pudieran comparar las variables estudiadas en grupos similares en cuanto a tamaño.

El grupo de estudiantes que realizaba bullying presentó una edad promedio de 13.8 años y estuvo integrado por 45 (55.6\%) hombres y 36 (44.4\%) mujeres. Por su parte, el grupo que no refirió dichas conductas presentó 13.6 años de edad promedio y en él se había 35 (43.2\%) hombres y 46 (56.8\%) mujeres.

\section{Instrumentos}

Se utilizaron dos instrumentos, el primero elaborado por Valdés et al. (2012), denominado Caracterización de la violencia escolar, que reportó validez de contenido a través de juicio de expertos y una confiabilidad medida a través del alfa de Cronbach de .89 .

Se identificó la presencia de bullying a través de una escala donde el estudiante tenía que responder acerca de la frecuencia con que llevó a cabo conductas violentas contra sus compañeros durante el último mes. Se le preguntó la frecuencia con la cual realizó durante el último mes este tipo de conductas, por ejemplo: patearlos, empujarlos, gritarles o excluirlos de actividades, entre otras, lo cual fue respondido utilizando una escala con cuatro opciones de respuesta: Nunca, Pocas veces (una vez al mes), Algunas veces (dos veces al mes) y Frecuentemente (más de dos veces al mes).

También se obtuvo información con respecto a variables de los estudiantes: a) desempeño académico, medido a través del promedio de calificaciones obtenidas por los estudiantes al terminar primaria y el del último bimestre de secundaria; b) problemas de disciplina; aquí se les preguntó: ¿fuiste reportado en primaria por problemas de disciplina?, ¿has sido reportado en secundaria por problemas de disciplina?, a lo que debían responder con una escala dicotómica "Sí" o "No"; c) abuso de sustancias, medido con la frecuencia con que reportaron consumo de alcohol y drogas durante el último mes y la frecuencia con la cual sus amigos cercanos han consumido alcohol y drogas durante el mismo periodo. Para responderlas se utilizó una escala con tres opciones de respuesta: Nunca, A veces y Frecuentemente, y d) pertenencia a bandas; se formularon dos preguntas: ¿perteneces a alguna banda juvenil?, ¿tus amigos cercanos pertenecen a bandas juveniles? El estudiante debía responder "Sí" o "No".

Con relación a la familia, mediante las respuestas al instrumento, se exploraron las variables: a) abuso de sustancias en los padres; se les cuestionó con respecto a la frecuencia con que sus padres consumen alcohol y drogas, a lo que respondieron con una escala tipo Likert con tres opciones de respuesta "Nunca", "A veces" y "Frecuentemente"; b) problemas legales de los padres; se les preguntó: ¿alguno de tus papás ha estado preso o ha tenido problemas con la ley?, a lo cual respondieron con "Sî" o "No", y c) estructura familiar; se les cuestionó: ¿vives con tus dos papas?, pregunta a la que contestaron con "Sí" o "No".

El segundo instrumento utilizado fue una adaptación realizada por Rascón (2012) del cuestionario Percepción de Clima Familiar de Moos, Moos \& Trickett (1984). La autora reportó obtener, a través de un análisis factorial con el método Oblimin y extracción de máxima verosimilitud, tres factores con 39 reactivos que explicaron el $50 \%$ de la varianza total de los puntajes. Estos factores los definió como: a) convivencia, funcionamiento familiar caracterizado por la presencia de relaciones, reglas y valores que favorecen el desarrollo del individuo; b) conflicto, presencia de situaciones desagradables y violencia en las relaciones familiares y c) estimulación del desarrollo, actividades que realizan la familia y/o sus integrantes y que favorecen el desarrollo. El instrumento evidenció confiabilidad medida a través del alfa de Cronbach de .90 y se contestó utilizando una escala tipo Likert que osciló de "Nunca" a "Siempre".

\section{Procedimiento}

Para la recolección de la información se solicitó la autorización informada de los directores y padres de familia. Posteriormente se les explicó a los estudiantes el objetivo del estudio y se les invitó a participar de manera voluntaria, garantizándoles la confidencialidad de los resultados.

El análisis de la información se realizó utilizando estadísticas descriptivas e inferenciales univariadas con el apoyo del software estadístico sPss. 20. 


\section{RESULTADOS}

Factores personales que diferencian a estudiantes con y sin conductas violentas en la escuela hacia los compañeros

Ajuste escolar. A través de una prueba t de Student para muestras independientes se compararon los promedios en primaria y secundaria de ambos grupos. Los resultados señalan que, si bien en el promedio de calificaciones de primaria no existieron diferencias significativas entre ambos grupos, el promedio de secundaria de los estudiantes que reportaron realizar bullying fue significativamente menor que el de los que no reportan tales conductas (ver tabla 1).

Posteriormente, a través de una prueba $\mathrm{X}^{2}$, se comparó la presencia de reportes por problemas de disciplina en primaria y secundaria de ambos grupos. Se apreció que en los dos niveles de estudio es significativamente mayor el número de estudiantes del grupo con reportes de bullying que refiere haber tenido este tipo de problemas en la escuela (ver tabla 2).

Ajuste psicosocial. A través de una prueba $\mathrm{X}^{2}$ se compararon las proporciones de estudiantes de ambos grupos que reportaron consumo de sustancias y pertenencia a bandas, ellos mismos y de amigos cercanos. Se apreció que existen más indicadores de dificultades de ajuste psicosocial en los estudiantes del grupo que reportó violencia hacia los compañeros, ya que tiene una mayor frecuencia de consumo de alcohol. Asimismo, los miembros de este grupo manifestaron que los amigos cercanos presentan una más elevada frecuencia de consumo de alcohol, drogas y pertenencia a bandas juveniles (ver tabla 3 ).

Tabla 1. Comparación de los promedios de calificaciones de los estudiantes con y sin reportes de bullying

\begin{tabular}{|c|c|c|c|c|c|}
\hline Promedios & Grupo & $x$ & $\mathrm{t}$ & gl & $\mathrm{p}$ \\
\hline \multirow{2}{*}{ Primaria } & Sin reportes de bullying & 89.4 & \multirow{2}{*}{.812} & \multirow{2}{*}{160} & \multirow{2}{*}{.416} \\
\hline & Con reportes de bullying & 88.4 & & & \\
\hline \multirow{2}{*}{ Secundaria } & Sin reportes de bullying & 85.1 & \multirow{2}{*}{2.362} & \multirow{2}{*}{160} & \multirow{2}{*}{$.019 *$} \\
\hline & Con reportes de bullying & 80.2 & & & \\
\hline
\end{tabular}

${ }^{*} \mathrm{p} \leq .05$

Tabla 2. Comparación de la frecuencia de reportes por problemas de disciplina en los grupos con y sin reporte de bullying

\begin{tabular}{|c|c|c|c|c|c|c|}
\hline \multirow{2}{*}{ Nivel } & & \multicolumn{2}{|c|}{ Reportes por problemas de disciplina } & \multirow{2}{*}{$\mathrm{X} 2$} & \multirow{2}{*}{ gl } & \multirow{2}{*}{$\mathrm{p}$} \\
\hline & & Grupo sin reporte de bullying & Grupo con reporte de bullying & & & \\
\hline \multirow{2}{*}{ Primaria } & No & 64 & 38 & \multirow{2}{*}{17.89} & \multirow{2}{*}{1} & \multirow{2}{*}{$.000^{*}$} \\
\hline & Sí & 17 & 43 & & & \\
\hline \multirow{2}{*}{ Secundaria } & No & 52 & 26 & \multirow{2}{*}{16.71} & \multirow{2}{*}{1} & \multirow{2}{*}{$.000^{*}$} \\
\hline & Sí & 29 & 55 & & & \\
\hline
\end{tabular}

${ }^{*} p \leq .05$ 
Tabla 3. Comparación de los grupos de estudiantes con y sin reportes de bullying en lo relativo a los indicadores de ajuste social

\begin{tabular}{|c|c|c|c|c|c|c|}
\hline \multicolumn{2}{|c|}{ Variables } & \multirow{2}{*}{$\begin{array}{c}\text { Frecuencia de } \\
\text { la conducta } \\
65\end{array}$} & \multirow{2}{*}{$\begin{array}{c}\begin{array}{c}\text { Frecuencia de } \\
\text { estudiantes por } \\
\text { grupos }\end{array} \\
41\end{array}$} & \multirow{2}{*}{$\begin{array}{c}X^{2} \\
15.79\end{array}$} & \multirow{2}{*}{$\begin{array}{r}\text { gl } \\
2\end{array}$} & \multirow{2}{*}{$\begin{array}{c}\mathbf{p} \\
.000^{*}\end{array}$} \\
\hline \multirow{3}{*}{ Consumo de alcohol } & Nunca & & & & & \\
\hline & $A$ veces & 13 & 31 & & & \\
\hline & Frecuentemente & 3 & 9 & & & \\
\hline \multirow{3}{*}{$\begin{array}{l}\text { Amigos con consumo } \\
\text { de alcohol }\end{array}$} & Nunca & 39 & 20 & 11.50 & 2 & $.000^{*}$ \\
\hline & A veces & 38 & 49 & & & \\
\hline & Frecuentemente & 4 & 12 & & & \\
\hline \multirow{3}{*}{ Consumo de drogas } & Nunca & 76 & 67 & 4.90 & 2 & .086 \\
\hline & A veces & 4 & 12 & & & \\
\hline & Frecuentemente & 1 & 2 & & & \\
\hline \multirow{3}{*}{$\begin{array}{l}\text { Amigos con consumos } \\
\text { de drogas }\end{array}$} & Nunca & 62 & 40 & 15.50 & 2 & $.000^{*}$ \\
\hline & A veces & 17 & 28 & & & \\
\hline & Frecuentemente & 2 & 13 & & & \\
\hline \multirow{2}{*}{ Pertenencia a bandas } & No & 72 & 65 & & 2 & \\
\hline & Sí & 9 & 16 & 2.31 & & 128 \\
\hline \multirow{2}{*}{$\begin{array}{l}\text { Amigos pertenecientes } \\
\text { a bandas }\end{array}$} & No & 65 & 54 & & 2 & \\
\hline & Sí & 16 & 27 & 3.83 & & $.037^{*}$ \\
\hline
\end{tabular}

${ }^{*} \mathrm{p} \leq .05$

Factores familiares que diferencian a estudiantes con y sin conductas violentas en la escuela hacia los compañeros

Composición familiar. Utilizando una prueba $\mathrm{X}^{2}$ se determinó que una mayor proporción de estudiantes del grupo que reportó bullying proviene de familias diferentes a la nuclear (ver tabla 4 ).

Ajuste psicosocial de la familia. A través de una prueba $\mathrm{X}^{2}$ se apreció que, de manera significativa, hay mayor frecuencia de consumo de drogas en los padres del grupo de estudiantes con reportes de bullying (ver tabla 5).

Clima familiar. Este apartado se evaluó a través de los tres factores del cuestionario de clima familiar: Convivencia, Conflicto y Estimulación del desarrollo. A través de una prueba $t$ de Student para muestras independientes se determinó que los miembros del grupo de estudiantes con conductas violentas reportan, de manera significativa, mayor presencia de conflictos en su familia (ver tabla 6). 
Tabla 4. Comparación de la composición familiar de los grupos de estudiantes con y sin reportes de bullying

\begin{tabular}{|c|c|c|c|c|c|}
\hline Tipo de familia & Grupo de estudiantes & Frecuencia & $x^{2}$ & gl & $\mathrm{p}$ \\
\hline \multirow{2}{*}{ Nuclear } & Sin reportes de bullying & 57 & \multirow[t]{4}{*}{5.84} & \multirow[t]{4}{*}{1} & \multirow{4}{*}{$.016^{*}$} \\
\hline & Con reportes de bullying & 24 & & & \\
\hline \multirow{2}{*}{ Diferente a la nuclear } & Sin reportes de bullying & 42 & & & \\
\hline & Con reportes de bullying & 39 & & & \\
\hline
\end{tabular}

${ }^{*} \mathrm{p} \leq .05$

Tabla 5. Comparación de los grupos de estudiantes con y sin reportes de bullying en lo relativo a los indicadores de ajuste psicosocial familiar

\begin{tabular}{|c|c|c|c|c|c|c|}
\hline \multirow[t]{2}{*}{ Variables } & \multirow[t]{2}{*}{ Frecuencia de la conducta } & \multicolumn{2}{|c|}{ Frecuencia de estudiantes por grupos } & \multirow[t]{2}{*}{$x^{2}$} & \multirow[t]{2}{*}{ gl } & \multirow[t]{2}{*}{$\mathrm{p}$} \\
\hline & & $\begin{array}{c}\text { Sin reportes de } \\
\text { bullying }\end{array}$ & $\begin{array}{c}\text { Con reportes de } \\
\text { bullying }\end{array}$ & & & \\
\hline $\begin{array}{l}\text { Consumo de alcohol } \\
\text { en padres }\end{array}$ & $\begin{array}{l}\text { Nunca } \\
\text { A veces } \\
\text { Frecuentemente }\end{array}$ & $\begin{array}{c}41 \\
38 \\
2\end{array}$ & $\begin{array}{c}40 \\
39 \\
2\end{array}$ & .025 & 2 & .987 \\
\hline $\begin{array}{l}\text { Consumo de drogas } \\
\text { en padres }\end{array}$ & $\begin{array}{l}\text { Nunca } \\
\text { A veces } \\
\text { Frecuentemente }\end{array}$ & $\begin{array}{c}74 \\
5 \\
2\end{array}$ & $\begin{array}{c}74 \\
2 \\
5\end{array}$ & 6.31 & 2 & $.043^{*}$ \\
\hline $\begin{array}{l}\text { Problemas legales en } \\
\text { los padres }\end{array}$ & $\begin{array}{l}\text { No } \\
\text { Sí }\end{array}$ & $\begin{array}{l}67 \\
14\end{array}$ & $\begin{array}{l}58 \\
23\end{array}$ & 2.83 & 2 & .092 \\
\hline
\end{tabular}

${ }^{*} p \leq .05$

Tabla 6. Comparación del clima familiar de los estudiantes con y sin reportes de bullying

\begin{tabular}{|c|c|c|c|c|c|}
\hline Factores & Grupo & $x$ & $\mathrm{t}$ & gl & $\mathrm{p}$ \\
\hline \multirow{2}{*}{ Convivencia } & Sin reportes de bullying & 4.84 & \multirow{2}{*}{.744} & \multirow{2}{*}{160} & \multirow{2}{*}{.458} \\
\hline & Con reportes de bullying & 4.72 & & & \\
\hline \multirow{2}{*}{ Conflicto } & Sin reportes de bullying & 2.96 & \multirow{2}{*}{-2.65} & \multirow{2}{*}{160} & \multirow{2}{*}{.009} \\
\hline & Con reportes de bullying & 3.37 & & & \\
\hline \multirow{2}{*}{$\begin{array}{l}\text { Estimulación del } \\
\text { desarrollo }\end{array}$} & Sin reportes de bullying & 3.80 & \multirow{2}{*}{-1.83} & \multirow{2}{*}{160} & \multirow{2}{*}{.069} \\
\hline & Con reportes de bullying & 3.43 & & & \\
\hline
\end{tabular}

${ }^{*} p \leq .05$ 


\section{DISCUSIÓN}

Los resultados indican que los estudiantes del grupo de secundaria que realizan bullying presentan mayores dificultades en el ajuste escolar que los del grupo sin tales conductas. Estas dificultades se expresan en circunstancias como menor desempeño académico y más problemas de disciplina en la escuela, mismas que han sido reportadas por otros estudios como características de estos estudiantes (Lozano \& García, 2000; Wegner, García, Nishimura \& Hishinuma, 2010). La presencia de estas dificultades de ajuste escolar corrobora lo expuesto por Ortega \& Del Rey (2008) y Pearce (2008), quienes sostienen que como parte de la prevención del fenómeno del bullying, es necesario desarrollar estrategias que promuevan una adecuada integración de los estudiantes al contexto escolar.

Por otra parte, se demostró que el grupo de estudiantes con reportes de bullying presentó mayores problemas de ajuste psicosocial, los cuales se evidenciaron por una mayor frecuencia de consumo de alcohol y de amigos con conducta de riesgo, como consumo de sustancias y pertenencia a bandas juveniles (Gentile et al., 2011; Houbre, Tarquino, Thuiller \& Hergot, 2006; Jankauskiene et al., 2008). Esto hace pensar que es urgente llevar a cabo intervenciones con estos estudiantes, ya que su vulnerabilidad va más allá del ambiente escolar, porque asumen comportamientos que aumentan el riesgo de presentar conductas delictivas y antisociales, lo que ha sido señalado como uno de los riesgos a mediano y largo plazo de los estudiantes agresores de los pares (Brendgen, Wanner, Vitaro, Bukowski \& Tremblay, 2007; Pearce, 2008).

Así mismo, se evidenció un ambiente familiar con mayor vulnerabilidad en el grupo de estudiantes que refirió efectuar bullying. Esta situación de riesgo se observó en mayor proporción dentro del grupo de estudiantes de familias diferentes a la nuclear, lo cual en muchas ocasiones afecta los recursos económicos y parentales de las familias (Ram \& Hou, 2003; Valdés,
Esquivel \& Artiles, 2007) y por ende, el funcionamiento familiar como tal, lo que puede influir negativamente en el desempeño académico y el ajuste socioemocional de los hijos (Valdés, Carlos, Urías \& Ibarra, 2011; Wallerstein, 1983).

También se evidenciaron otros indicadores de disfuncionalidad en las familias de los estudiantes que tienen conductas de bullying, como mayor frecuencia de consumo de drogas entre los padres y un clima familiar caracterizado por el conflicto. Estos aspectos se asocian con la violencia en los hijos, ya que, por una parte, los padres se convierten en modelos sociales negativos y por otra, dichas conductas dificultan la comunicación y el apoyo que le pueden brindar a sus hijos (Cava et al., 2006).

\section{CONCLUSIONES}

Los hallazgos del presente estudio afirman la conveniencia de abordar el fenómeno del bullying desde una perspectiva ecológica y sistémica (Coloroso, 2004; Swearer et al., 2009). Esto se corroboró en la existencia de variables que diferencian al grupo de estudiantes con conductas violentas ubicadas en diferentes sistemas, en este caso el individual y el familiar. El hallazgo anterior sugiere, además, la necesidad de que los programas de prevención de violencia escolar realicen acciones dirigidas a contrarrestar los factores de riesgo ubicados en estos niveles de funcionamiento, involucrando tanto aspectos de los propios estudiantes como de sus familias (Merrell, Gueldner, Ross \& Isava, 2008; Tutty, 2008).

Es necesario establecer ciertas limitaciones de la presente investigación: primero, no haber considerado las posibles interacciones en las variables integradas en el estudio, y segundo, no tener en cuenta variables relativas a la escuela y contexto social del estudiante, que según se ha reconocido en otros estudios, contribuyen a la explicación de la violencia entre pares (Ahmed \& Braithwaite, 2004; Ortega \& Del Rey, 2008). 


\section{REFERENCIAS}

Ahmed, E. \& Braithwaite, V. (2004). "What, me ashamed?" Shame management and school. Journal of Research Crime and Delinquency, 41(3), 269-294. doi: 10.1177/0022427804266547.

Avilés, J. \& Monjas, I. (2005). Estudio de la incidencia de la intimidación y el maltrato entre iguales en la educación secundaria obligatoria mediante el cuestionario (CIMEI). Anales de Psicología, 21(1), 27-41.

Besag, V. (2008). Padres y maestros trabajando juntos. En M. Elliot (Ed.), Intimidación. Una guía práctica para combatir el miedo en las escuelas (pp. 219238). Distrito Federal, México: Fondo de Cultura Económica.

Brendgen, M., Wanner, B., Vitaro, F., Bukowski, W. \& Tremblay, R. (2007). Verbal abuse by the teacher during childhood and academic, behavioral, and emotional adjustment in young adulthood. Journal of Educational Psychology, 99(1), 26-38. doi: 10.1037/0022-0663.99.126.

Bringas, C., Rodríguez, F. \& Herrero, F. (2009). Responsabilidad y comportamiento antisocial del adolescente como factores asociados al rendimiento escolar. Acta Colombiana de Psicología, 12(2), 69-76.

Cassidy, T. (2009). Bullying and victimization in school children: The role of social identity, problem-solving style, and family and school context. Social Psychology of Education, 12, 63-76. doi: 10.1007/ s11218-008-9016-y.

Castillo, C. \& Pacheco, M. (2008). Perfil del maltrato entre estudiantes de secundaria en la ciudad de Mérida, Yucatán. Revista Mexicana de Investigación Educativa, 13(8), 825-848.

Cava, M., Musitu, G. \& Murgui, S. (2006). Familia y violencia escolar: el rol mediador de la autoestima y la actitud hacia la autoridad institucional. Psicothema, 18(003), 367-373.

Coloroso, B. (2004). The bully, the bullied and the bystander. New York, usa: Harper Colling.

Díaz, M. (2005). Por qué se produce la violencia y cómo prevenirla. Revista Iberoamericana de Educación, 37, 17-47.

Eiden, R., Ostrov, J., Colden, C., Leonard, K., Edwards, E. \& Orrange-Torchia, T. (2010). Parent alcohol problems and peer bullying and victimization: child gender and toddler attachment security as moderators.
Journal of Clinical Child \& Adolescent Psychology, 39(3), 341-350. doi: 10.1080/15374411003691768.

Estévez, E., Martínez, B., Moreno, D. \& Musitu, G. (2006). Relaciones familiares, rechazo entre iguales y violencia escolar. Cultura y Educación, 18(3-4), 335-344.

Estévez, E., Murgui, S., Moreno, D. \& Musitu, G. (2007). Estilos de comunicación familiar, actitud hacia la autoridad institucional y conducta violenta del adolescente en la escuela. Psichotema, 19(1), 108-113.

Gentile, D., Coyne, S. \& Walsh, D. (2011). Media violence, physical aggression, and relational aggression in school age children: a short-term longitudinal study. Aggressive Behavior 37, 193-206. doi: 10.1002/ ab. 20380 .

Houbre, B., Tarquino, C., Thuiller, I. \& Hergot, E. (2006). Bullying among students and its consequences on health. European Journal of Psychology of Education, XXI(2), 183-202.

Instituto Nacional de Evaluación Educativa (2006). Informe sobre calidad de la Educación Básica. Violencia $y$ disciplina en escuelas primarias y secundarias. Distrito Federal, México: INEE.

Jankauskiene, R., Kardelis, K., Sukys, S. \& Kardeliene, L. (2008). Association between school bullying and psychosocial factor. Social Behavior and Personality, 36(2), 145-162.

Kelly, J. \& Emery, R. (2003). Children adjustment following divorce: risk and resilience perspective. Family Relations, 52, 352-362. doi: 10.1111/j.1741-3729.2003. 00352.X.

Lozano, L. \& García, L. (2000). El rendimiento académico y los trastornos emocionales y del comportamiento. Psicothema, 12(2), 340-343.

McWhirter, J., McWhirter, B., McWhirter, A. \& McWhirter, E. (1993). At-risk youth: A comprehensive response. Belmont, CA, UsA: Thomson Brooks/Cole Publishing.

Merrell, K., Gueldner, B., Ross, W. \& Isava, D. (2008). How effective are school bullying intervention programs? A meta-analysis of intervention research. School Psychology Quarterly, 23(1), 26-42. doi: 10.1037/1045-3830.23.1.26

Moos, R., Moos, B. \& Trickett, E. (1984). FES, WES, CIES, CES. Escalas de Clima Social. Madrid, España: TEA.

Morales, H. (2008). Factores asociados y trayectorias del desarrollo del comportamiento antisocial durante 
la adolescencia. Implicaciones para la prevención. Revista Interamericana de Psicología, 42(1), 129-142.

Muñoz, G. (2008). Violencia escolar en México y otros países. Comparaciones a partir de los resultados del Instituto Nacional de Educación. Revista Mexicana de Investigación Educativa, 13(39), 1195-1228.

Nansel, T., Overpeck, M., Pilla, R., Ruan, W., SimonsMorton, B. \& Scheidth, P. (2001). Bullying behaviors among US youth. Prevalence and association with psychosocial adjustment. JAMA, 285(16), 20942100. doi: 10.1001/jama.285.16.2094.

Olweus, D. (1993). Bullying at school. Victoria, Australia: Blackwell Publishing.

Ortega, R. \& Del Rey, R. (2008). La violencia escolar. Estrategias de prevención (4ta. Ed.). Barcelona, España: Grao.

Ozer, A., Tarik, T. \& Atik, G. (2011). Individual correlates of bullying behavior in Turkish middle schools. Australian Journal Guidance and Counseling, 21(21), 186-202. doi: 10.3775/ajgc.21.2.186.

Pearce, J. (2008). ¿Qué se puede hacer con el agresor? En M. Elliot (Ed.), Intimidación. Una guía práctica para combatir el miedo en las escuelas (pp. 157-186). Distrito Federal, México: Fondo de Cultura Económica.

Ram, B. \& Hou, F. (2003). Changes in family structure and child outcomes: roles of Economic and familiar resources. The Policy Studies Journal, 31(3), 309-330. doi: 10.1111/1541-0072.00024.

Rascón, K. (2012). Propiedades psicométricas de un cuestionario para medir clima familiar en estudiantes de secundaria. (Tesis de licenciatura no publicada). Instituto Tecnológico de Sonora. Sonora, México.

Sánchez, P. \& Valdés, A. (2003). El menor infractor: una categoría infundada en el sistema de educación especial en México. Perfiles Educativos, xxv(099), 72-90.

Stephenson, P. \& Smith, D. (2008). ¿Por qué algunas escuelas no tienen acosadores? En M. Elliot (Ed.), Intimidación. Una guía práctica para combatir el miedo en las escuelas (pp. 56-78). Distrito Federal, México: Fondo de Cultura Económica.

Swearer, S., Espegale, D. \& Napolitano, S. (2009). Bullying. Prevention \& Intervention. Realistic Strategies for Schools. New York, USA: The Guilford Press.

Turagabeci, A., Nakamura, K. \& Takano, T. (2008). Healthy lifestyle Behavior decreasing risk of being bullied, violence and injury. Plos One, 3(2), 1-7. doi: 10.1371/journal.pone.0001585.
Tutty, L. (2008). Best practices in school-based bullying prevention programs: What works? En Debra Pepler \& Wendy Craig (Eds.), Understanding and addressing bullying: An International Perspective (pp. 144-163). USA: Authothouse.

Valdés, A. (2010). La familia ante el divorcio o la separación. En A. Valdés \& J. Ochoa (Eds.), Familia y crisis. Estrategias de afrontamiento (pp. 139-152). Distrito Federal, México: Pearson.

Valdés, A., Carlos, E., Urías, M. \& Ibarra, B. (2011). Efectos del divorcio de los padres en el desempeño académico y la conducta de los hijos. Enseñanza e Investigación en Psicología, 16(2), 295-308.

Valdés, A., Esquivel, L. \& Artiles, K. (2007). Familia y desarrollo. Estrategias de intervención en terapia familiar. Distrito Federal, México: El Manual Moderno.

Valdés, A., Ojeda, A., Urías, M. \& Ponce, D. (en prensa). Factores de riesgo en estudiantes de secundaria con conductas violentas hacia los compañeros. En J. Vales, A. Valdés, J. Angulo, C. García \& I. Castro (Eds.), Investigación Psicoeducativa en Sonora. Resultados y propuestas de acción. Distrito Federal, México: Pearson.

Valdés, A., Urías, M., Tapia, C., Torres, G., Carlos, E., Vera, J., Montoya, G. (2012). Caracterización de la violencia escolar en las escuelas secundarias públicas del sur de Sonora. Reporte Técnico. Proyecto financiado por PROMEP, Sonora, México, ITSON.

Villareal, M., Sánchez, J. \& Gonzalo, M. (2010). Un modelo predictivo de la violencia escolar en adolescentes. Ciencias UANL, XII(4), 413-420. Recuperado en redalyc.uaemex.mx/pdf/402/40215505012.pdf.

Wallerstein, J. (1983). Children of Divorce: Stress and Developmental Task. Nueva York, USA: McGraw-Hill.

Wegner, E., García, O., Nishimura, S. \& Hishinuma, E. (2010). Educational performance and attitudes towards school as risk-protective factors for violence: a study of the Asian/Pacific Islander Youth Violence Prevention Center. Psychology in the Schools, 47(8), 789-802. doi: 10.1002/pits.20504.

White, N. \& Loeber, R. (2008). Bullying and special education as predictor of serious delinquency. Journal of Research in Crime and Delinquency, 5(4), 380-397. doi: $10.1177 / 0022427808322612$. 